\title{
The Expression of Carbonic Anhydrase (CA) IX/XII and Lymph Node Metastasis in Early Breast Cancer
}

\author{
Keun-Yong Eom, MD, PhD ${ }^{1}$ \\ Min Hye Jang, MD² \\ So Yeon Park, MD, $\mathrm{PhD}{ }^{2}$ \\ Eun Young Kang, $\mathrm{MD}^{3}$ \\ Sung Won Kim, MD, PhD 3 \\ Jee Hyun Kim, MD, PhD ${ }^{3}$ \\ Jae-Sung Kim, MD, PhD \\ In Ah Kim, MD, PhD ${ }^{1,4}$
}

Departments of ${ }^{1}$ Radiation Oncology and

${ }^{2}$ Pathology, ${ }^{3}$ Breast Care Center,

Seoul National University Bundang Hospital,

Seoul National University

College of Medicine, Seongnam,

${ }^{4}$ Medical Science Research Institute,

Seoul National University Bundang Hospital,

Seongnam, Korea

\author{
Correspondence: In Ah Kim, MD, PhD \\ Department of Radiation Oncology, \\ Seoul National University Bundang Hospital, \\ 82 Gumi-ro 173 beon-gil, Bundang-gu, \\ Seongnam 13620, Korea \\ Tel: 82-31-787-7651 \\ Fax: 82-31-787-4019 \\ E-mail: inah228@snu.ac.kr \\ Received August 27, 2014 \\ Accepted December 10, 2014 \\ Published Online March 3, 2015 \\ *Keun-Yong Eom and Min Hye Jang \\ contributed equally to this work.
}

\section{Purpose}

The aim of study was to test by immunohistochemical $(\mathrm{IHC})$ staining whether carbonic anhydrase (CA) 9 and 12 have an effect on sentinel lymph node (SLN) metastasis in early breast cancer and to find clinicopathologic factors associated with SLN metastasis.

\section{Materials and Methods}

Between June 2003 and June 2011, medical records of 470 patients diagnosed with breast cancer with pT1-2, pNO-2, and MO were reviewed. Of these 470, 314 patients who underwent SLN biopsyıaxillary dissection were subjects of this study. Using tissue microarray, IHC staining for CA9 and CA12 was performed. Clinicopathologic factors such as patient age, tumour size, lymphatic invasion, hormone receptor status, and the Ki-67 labeling index were analysed together.

\section{Results}

The mean age of all patients was 51.7 years. The mean number of harvested SLN was 3.62, and 212 patients (67.5\%) had negative SLN. Lymphatic invasion, the Ki-67 labelling index of primary tumours, and CA9 staining of stromal cells, were independent risk factors for SLN metastasis in the multivariate analysis. In 33 patients (10.5\%) without the three risk factors, no patient had SLN metastasis. In 80 patients without lymphatic invasion of primary tumours or CA9 staining of stromal cells, only four patients (5\%) had positive SLN.

\section{Conclusion}

CA9 staining of stromal cells is an independent risk factor for SLN metastasis as well as lymphatic invasion and a low Ki-67 labelling index of primary tumours in patients with early breast cancer. IHC staining of primary tumours for CA12 was not associated with SLN metastasis.

\section{Introduction}

Lymph node status of breast cancer is the single most important prognostic factor for overall survival. The incidence of lymph node metastases in small breast cancer is reported to be in the range of 20\%-30\% [1,2]. Younger age, negative hormone receptor status, and presence of lymphovascular invasion are associated with lymph node metastasis

\section{Key words}

Breast neoplasms, Carbonic anhydrase 9, Carbonic anhydrase 12, Sentinel lymph node metastases, Stromal cells 
tumour-associated cell migration and invasion [8]. Expression of CA9 is a poor prognostic factor for breast cancer [9].

The authors hypothesized that hypoxia affects early breast cancer, specifically in sentinel lymph node (SLN) metastasis. As the relationship between SLN metastasis and the expression of CA9 and CA12 in early breast cancer is uncertain, we investigated whether the expression of CA9 and CA12 of tumours and stromal cells is associated with SLN metastasis in patients with early breast cancer. At the same time, patient age, tumour size, multiplicity, histologic grade, lymphatic and venous invasion, hormone receptor status, human epidermal growth factor receptor 2 (HER2) status, Ki-67 labeling index were analysed. The data in this report follows the reporting recommendations for tumour marker prognostic studies (remark) [10].

\section{Materials and Methods}

Patients diagnosed between June 2003 and June 2011 with breast cancer with pT1-2, pN0-N2 and without distant metastases were enrolled into this retrospective study. Patients who underwent neoadjuvant chemotherapy were excluded. Through the institutional database, 475 patients who had pathologic specimens available for immunohistochemical (IHC) assay of CA9 and CA12 were found, and of these, the 314 patients receiving SLN biopsy \pm axillary lymph node dissection were the subjects of this study. This study was approved by the Institutional Review Board and informed consent was waived.

All patients were preoperatively evaluated with physical examinations, breast ultrasonography, breast magnetic resonance imaging, and chest computed tomography. Breast conserving surgery or mastectomy was performed by two dedicated breast surgeons, and pathologic examination of SLN was done using serial section by two specialised pathologists.

Patient age, tumour size, multiplicity, histologic grade, presence of ductal carcinoma in situ, necrosis, lymphatic and venous invasion, hormone receptor status, HER2, epidermal growth factor receptor, $\mathrm{Ki}-67$, and the number of positive lymph nodes along with harvested sentinel nodes were collected by reviewing medical records. The baseline characteristics are summarised in Table 1.

\section{Tissue microarray construction}

We constructed tissue microarray (TMA) blocks using the 314 cases of invasive breast cancers (IBCs). To reduce sampling errors in TMA evaluation, all hematoxylin and eosin
Table 1. Clinicopathologic characteristics of patients

\begin{tabular}{|c|c|}
\hline Characteristic & No. $(\%)(n=314)$ \\
\hline Mean age (range, yr) & $51.7(25.6-86.7)$ \\
\hline Mean tumour size (range, $\mathrm{cm}$ ) & $2.05(0.5-4.8)$ \\
\hline \multicolumn{2}{|l|}{ T stage } \\
\hline $1 \mathrm{a}$ & $1(0.3)$ \\
\hline $1 \mathrm{~b}$ & $19(6.1)$ \\
\hline $1 \mathrm{c}$ & $162(51.6)$ \\
\hline 2 & $130(42.0)$ \\
\hline \multicolumn{2}{|l|}{ Histologic type } \\
\hline Invasive duct & $274(87.3)$ \\
\hline Invasive lobular & $11(3.5)$ \\
\hline Mucinous & $10(3.2)$ \\
\hline Others & $19(6.1)$ \\
\hline \multicolumn{2}{|l|}{ Multiplicity } \\
\hline No & $258(82.2)$ \\
\hline Yes & $56(17.8)$ \\
\hline \multicolumn{2}{|l|}{ Histologic grade } \\
\hline I & $56(17.8)$ \\
\hline II & $96(30.6)$ \\
\hline III & $162(51.6)$ \\
\hline \multicolumn{2}{|l|}{ Lymphatic invasion } \\
\hline No & $193(61.5)$ \\
\hline Yes & $121(38.5)$ \\
\hline \multicolumn{2}{|l|}{ Venous invasion } \\
\hline No & $303(96.5)$ \\
\hline Yes & $11(3.5)$ \\
\hline \multicolumn{2}{|l|}{ Estrogen receptor } \\
\hline Negative & $84(26.8)$ \\
\hline Positive & $230(73.2)$ \\
\hline \multicolumn{2}{|l|}{ Progesterone receptor } \\
\hline Negative & $101(32.2)$ \\
\hline Positive & $199(63.4)$ \\
\hline \multicolumn{2}{|l|}{ HER2 } \\
\hline Negative & $263(83.8)$ \\
\hline Positive & $51(16.2)$ \\
\hline \multicolumn{2}{|l|}{ Ki-67 } \\
\hline$<20 \%$ & $182(58.0)$ \\
\hline$\geq 20 \%$ & $132(42.0)$ \\
\hline \multicolumn{2}{|l|}{ N stage } \\
\hline 0 & $212(67.5)$ \\
\hline 1 & $89(28.3)$ \\
\hline 2 & $13(4.1)$ \\
\hline \multicolumn{2}{|l|}{ Breast surgery } \\
\hline Lumpectomy & $213(67.8)$ \\
\hline Mastectomy & $101(32.2)$ \\
\hline \multicolumn{2}{|l|}{ Axillary surgery } \\
\hline Sentinel Bx & $209(66.6)$ \\
\hline Sentinel Bx+ALND & $105(33.4)$ \\
\hline Mean sentinel LNs (range) & $3.62(1-10)$ \\
\hline
\end{tabular}

Bx, biopsy; ALND, axillary lymph node dissection; LN, lymph node. 

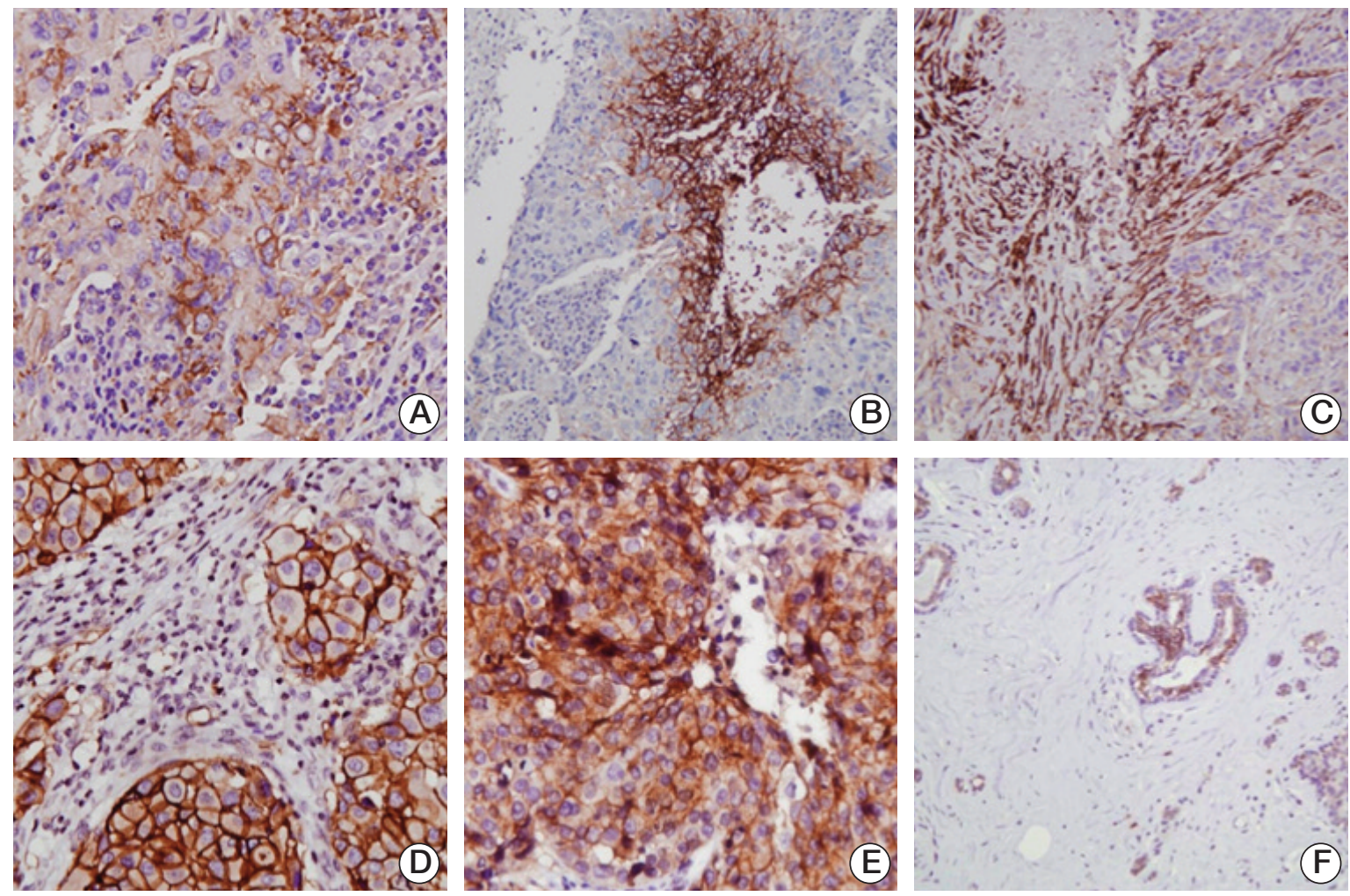

Fig. 1. Representative photos of carbonic anhydrase (CA) 9 and CA12. (A) strong membranous staining of CA9 is observed in tumour cells $(\times 200)$. (B) Expression of CA9 is frequently observed around the intratumoural necrosis $(\times 100)$. (C) CA9 is expressed in stromal fibroblast as well as tumour cells $(\times 100)$. (D) Strong membranous staining of CA12 is observed $(\times 200)$. (E) Diffuse cytoplasmic staining of CA12 is observed with moderate intensity $(\times 200)$. (F) CA12 expression is identified in normal ductal epithelium especially in luminal cells $(\times 100)$.

stained slides were reviewed and the most representative tumour section was selected for each case. Three tissue columns of invasive carcinoma of $2.0 \mathrm{~mm}$ in diameter were taken from different areas of the tumours and arranged in new TMA blocks using a trephine apparatus (Superbiochips Laboratories, Seoul, Korea). In each TMA block, two columns of normal breast tissue were included for negative controls.

\section{IHC staining and scoring}

The expression of standard biomarkers including estrogen receptor (ER), progesterone receptor (PR), HER2, and Ki-67 were evaluated in whole sections at the time of diagnosis or in in TMA sections for missing data during the study. Tissue sections $4 \mu \mathrm{m}$ thick were cut, dried, deparaffinised, and rehydrated following standard procedures. All of the sections were subjected to heat-induced antigen retrieval. IHC staining was carried out using TMAs in a BechMark XT autostainer (Ventana Medical Systems, Tucson, AZ), using an i-View detection kit (Ventana Medical Systems) for ER (1:100, clone SP1, LabVision, Fremont, CA), PR (1:70, PgR 636, Dako, Glostrup, Denmark), HER2 (1:700, polyclonal, Dako), Ki-67 (1:250, MIB-1, Dako), CA9 (1:200, ab15086, Abcam, Cambridge, UK), and CA12 (1:100, ab140385, Abcam). ER and PR were regarded as positive if there were at least $1 \%$ positive tumour cells. Expression of HER2 was scored according to the American Society of Clinical Oncology/College of American Pathologists guidelines [11,12]. HER2 IHC3+ or amplification in fluorescence in situ hybridization was regarded as HER2 positive. For the Ki-67 proliferation index, cases with $20 \%$ or more positive tumour cells were regarded as having high indices. For CA9, membranous staining of tumour cells was examined in a semi-quantitative way. The staining intensity of tumour cells was scored from 0 to 3 ( 0 , no staining; 1 , weak; 2 , moderate; and 3 , strong) as did the percentage of positive tumour cells from 0 to 100 . We also evaluated staining of stromal cells around the tumour cell nests. For CA12, the cytoplasmic and mem- 
Table 2. Pearson's correlation and logistic regression analyses of predictive factors for lymph node metastases

\begin{tabular}{|c|c|c|c|c|c|}
\hline \multirow{2}{*}{ Variable } & \multicolumn{2}{|c|}{ Univariate analysis } & \multicolumn{3}{|c|}{ Multivariate analysis } \\
\hline & Spearman's $\rho$ & p-value & Adjusted OR & $95 \% \mathrm{CI}$ & p-value \\
\hline Age & -0.144 & 0.010 & - & - & - \\
\hline Tumour size & 0.100 & 0.078 & - & - & - \\
\hline T stage & 0.107 & 0.058 & - & - & - \\
\hline Multiplicity & 0.068 & 0.232 & - & - & - \\
\hline Histologic grade & -0.131 & 0.021 & - & - & - \\
\hline Lymphatic invasion & 0.331 & $<0.001$ & 4.613 & $2.736-7.778$ & $<0.001$ \\
\hline Venous invasion & 0.164 & 0.004 & - & - & - \\
\hline Estrogen receptor & 0.112 & 0.047 & - & - & - \\
\hline Progesterone receptor & 0.135 & 0.019 & - & - & - \\
\hline Ki- $67 \geq 20 \%$ & -0.136 & 0.016 & 0.551 & $0.323-0.938$ & 0.028 \\
\hline MembCA12 & 0.188 & 0.001 & - & - & - \\
\hline CytoCA12 & 0.165 & 0.004 & - & - & - \\
\hline StromaCA9 & 0.187 & 0.001 & 2.601 & $1.506-4.492$ & 0.001 \\
\hline
\end{tabular}

OR, odds ratio; $\mathrm{CI}$, confidence interval; MembCA12, membrane staining by carbonic anhydrase (CA) 12; CytoCA12, cytoplasmic staining by CA12; StromaCA9, staining by CA9 in stromal cells.

branous staining of tumour cells was evaluated separately in the same way for CA9. Any positive staining in the membrane or cytoplasm was regarded as positive.

\section{Statistical analyses}

All cases with at least one available TMA for IHC were used for statistical analyses. If there was any difference in IHC staining results between three cores, we chose the results from the largest one as representative and used them for analyses. Statistical analysis used SPSS ver. 20 (IBM Co., Chicago, IL). Null hypotheses of no difference were rejected if p-values were less than 0.05 , or, equivalently, if the $95 \%$ confidence intervals (CIs) of risk point estimates excluded 1. Categorical data were compared using Fisher exact test or Pearson's chi-square test, while continuous data were compared using t test. To determine a cut-off value for Ki-67, receiver operating curve was used. Correlation between variables was evaluated using Spearman's correlation coefficient. Multivariate binary logistic regression model was applied to determine significant factors for SLN metastasis.

\section{Results}

The mean age was 51.7 years, ranging from 25.6 to 86.7 years, and the mean tumour size was $2.05 \mathrm{~cm}$ (Table 1). Most patients $(87.3 \%$ ) had invasive duct carcinoma, and 56 patients $(17.8 \%)$ had multiple disease. About half of the patients $(51.6 \%)$ had high histologic grade with lymphatic invasion of tumours in $38.5 \%$. ER positivity was $73.2 \%$ and HER2 was $16.2 \%$. The N stage of patients was N0 in 212 patients $(67.5 \%), \mathrm{N} 1$ in $89(28.3 \%)$, and $\mathrm{N} 2$ in $13(4.1 \%)$. Breast conserving surgery was performed in 213 patients $(67.8 \%)$ and SLN biopsy only in 209 (66.6\%). The mean number of harvested SLN was 3.62, ranging from 1 to 10.

\section{IHC expression of CA9 and CA12}

CA9 expression was observed both in tumour cells and stromal cells. CA9 was expressed predominantly around the tumour cell membrane with various amounts of cytoplasmic staining. Of 314 IBCs, 191 cases $(60.8 \%)$ showed CA9 expression in tumour cells. CA9 expression was strong around the intratumoural necrosis and its intensity was correlated with the presence of intratumoural necrosis $(p<0.001)$. Peritumoural stromal cells also expressed membranous and cytoplasmic CA9. One hundred and eighty-three cases (58.3\%) expressed CA9 in pertitumoural stromal cells with various staining intensities.

CA12 expression was 71.0\% (223/314) in tumor cell membrane and $73.9 \%$ (232/314) in cytoplasm of tumour cells. As for stromal cells, only 57 of 312 cases $(18.3 \%)$ showed stromal expression of CA12, excluding two patients whose TMA cores did not contain enough peritumoural stroma. CA12 was frequently expres-sed in normal ductal epithelium with weak intensity (Fig. 1). 
Table 3. Correlation of risk score 1 with $\mathrm{N}$ stage

\begin{tabular}{|c|c|c|c|c|c|}
\hline \multirow{2}{*}{ N stage } & \multicolumn{4}{|c|}{ Risk score $1^{\text {a) }}$} & \multirow{2}{*}{ Total } \\
\hline & 0 & 1 & 2 & 3 & \\
\hline 0 & $33(10.5)$ & 93 (29.6) & $68(21.7)$ & $18(5.7)$ & $212(67.5)$ \\
\hline 1 & 0 & $25(8.0)$ & 42 (13.4) & $22(7.0)$ & 89 (28.3) \\
\hline 2 & 0 & $2(0.6)$ & $5(1.6)$ & $6(1.9)$ & $13(4.1)$ \\
\hline Total & $33(10.5)$ & $120(38.2)$ & $115(36.6)$ & 46 (14.6) & $314(100)$ \\
\hline
\end{tabular}

a) Risk score 1: number of risk factors (lymphatic invasion and Ki-67 < 20\% of primary tumours, and carbonic anhydrase 9 expression in stromal fibroblasts).

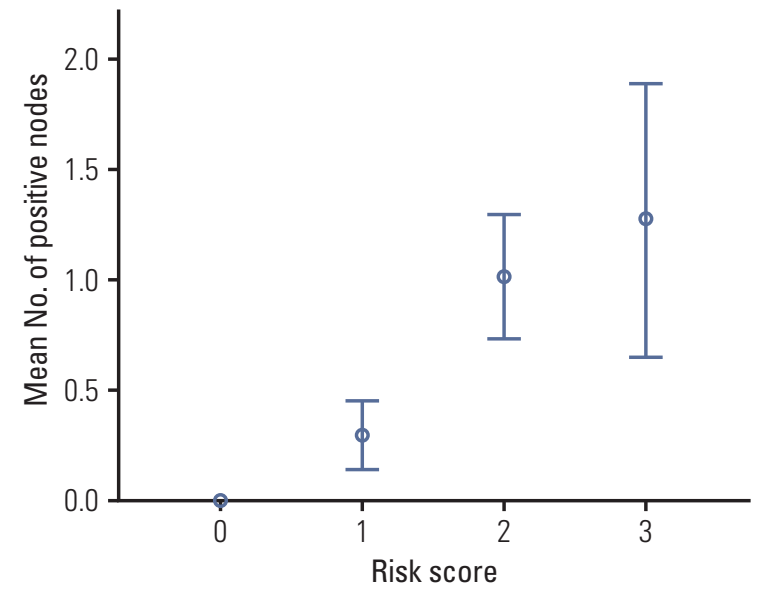

Fig. 2. The mean number of positive lymph nodes according to risk score 1 . The mean number of positive lymph nodes increases proportional to risk score, and was different between groups with statistical significance $(p<0.05)$ except between risk scores 2 and 3. Bar indicates the standard error.

\section{Risk factors for SLN metastases}

In the univariate analysis, several clinicopathologic conditions were positively related to SLN metastases. Those are young patient age, presence of lymphatic and venous invasion, ER or PR positivity, a low Ki-67 labeling index, positive staining of membrane and cytoplasm of tumours for CA12, and CA9 staining of stromal cells (Table 2).

Among these factors, lymphatic invasion and the Ki-67 labelling index of primary tumours, and CA9 expression of stromal cells were significant risk factors in multivariate analysis. The adjusted odds ratio (OR) of the presence of lymphatic invasion was 4.613, with a 95\% CI 2.736-7.778 when compared with the absence of lymphatic invasion. The OR of CA9 staining in stromal cells was 2.601 (95\% CI, 1.506 to
4.492); that of Ki-67 $\geq 20 \%$ of primary tumours, $0.551(95 \%$ CI, 0.323 to 0.938 ).

In 121 patients with lymphatic invasion of primary tumours, 63 (52.1\%) had SLN metastasis. Lymphatic invasion of primary tumours was also associated with younger age, smaller tumour size, and IHC membrane staining of CA12 ( $\mathrm{p}<0.001, \mathrm{p}=0.026$, and $\mathrm{p}<0.001$, respectively). In 189 patients with CA9 positive stromal cells, $73(38.6 \%)$ had SLN metastasis. As for Ki-67, 28 of 132 patients (21.2\%) with Ki-67 $\geq 20 \%$ had SLN metastasis. In 182 patients with Ki-67 $<20 \%, 69(37.9 \%)$ had SLN metastasis. Ki-67 $\geq 20 \%$ was asso ciated with a high histologic grade, high mitotic count, and ER negativity $(\mathrm{p}<0.001, \mathrm{p}=0.004$, and $\mathrm{p}<0.001$, respectively).

\section{A model predicting SLN metastases}

A risk score model (RSM) predicting SLN metastasis was created using significant predictors in multivariate analysis. First, four tiered risk groups (RSM1) were generated, counting the three risk factors including lymphatic invasion and a low Ki-67 labelling index of primary tumours, and CA9 positivity of stromal cells. Table 3 shows the relationship of RSM1 and N stage. In 33 patients without risk factors, no patient had SLN metastasis, and the percentage of patients having SLN metastasis increased proportional to the score of RSM1 ( $\mathrm{p}<0.001)$. The mean number of positive lymph nodes also increased proportional to that of RSM1 (Fig. 2).

Second, RSM2, including lymphatic invasion of primary tumours and CA9 positivity of stromal cells as risk factors, was evaluated. In 80 patients without the two risk factors, only four patients (5\%) had positive SLN. RSM2 was statistically significant as well as RSM1 ( $<0.001)$. 
Table 4. Correlation of risk score 2 with $\mathrm{N}$ stage

\begin{tabular}{lcccc} 
& \multicolumn{3}{c}{ Risk score $\mathbf{2}^{\mathrm{a}} \mathbf{2}$} & \multirow{2}{*}{ Total } \\
\cline { 2 - 4 } & $\mathbf{0}$ & $\mathbf{1}$ & $\mathbf{2}$ & $212(67.5)$ \\
0 & $76(35.8)$ & $104(49.1)$ & $32(15.1)$ & $89(28.3)$ \\
1 & $4(1.3)$ & $54(17.2)$ & $31(9.9)$ & $13(4.1)$ \\
2 & 0 & $6(1.9)$ & $7(2.2)$ & $314(100)$ \\
\hline
\end{tabular}

a)Risk score 2: number of risk factors including lymphatic invasion of primary tumours and carbonic anhydrase 9 expression of stromal fibroblasts.

\section{Discussion}

We evaluated the relationship between SLN metastasis and the expression of CA9 and CA12 in patients with early breast cancer. In this study, positive CA9 staining of stromal cells was strongly associated with SLN metastasis as well as lymphatic invasion of primary tumours, whereas CA12 was not.

CA9 is related to tumour hypoxia and is a marker for poor survival of patients with advanced breast cancer [13]. This implicates tumour hypoxia as one condition that potentiates metastasis of tumours. Several studies observed that CA9 expression in tumour cells is relevant to lymph node metastasis. Aomatsu et al. [14] showed that the CA9 expression in a core needle biopsy specimen of breast cancer was significantly associated with lymph node metastasis (70\% vs. 30\%) and lymphovascular invasion (69\% vs. 31\%). Schutze et al. [15] investigated CA9 mRNA signalling intensity using TMA and observed that a high CA9 expression was associated with positive nodal status as well as with shorter disease-free survival. However, little is known about the relationship between CA9 expression in stromal cells around tumour cells and lymph node metastasis. In this study, CA9 staining of stromal cells was statistically significantly correlated with SLN metastases, as well as lymphatic invasion of primary tumours. This finding is novel in IBCs, suggesting that CA9 expression of stromal cells is more important for SLN metastasis than for primary tumours. Brockton et al. [16] reported similar observations in head and neck cancer. They showed that in head and neck cancer cohorts, stromal CA9 levels are more strongly associated with both poor survival and lymph node metastasis than tumour CA9. Fiaschi et al. [17] found that CA9 from cancer-associated fibroblasts drives epithelialmesenchymal transition in prostate cancer, a key event associated with increased motility.

CA12 is an enzyme that catalyses the hydration of carbon dioxide to bicarbonate and protons. CA12 is up-regulated by hypoxia via hypoxia-inducible factor-1, although the under- lying mechanism is not clear. In a study using a breast cancer cell line, the expression of CA12 was related to the invasion and migration ability of breast cancer cells [18]. In contrast to in vitro data, CA12 expression is highly associated with ER [19] and is an indicator of a good prognosis in patients with breast cancer [20]. Watson et al. [20] reported that the expression of CA12 was associated with lower grade, positive ER, negative epidermal growth factor receptor, and the absence of necrosis. In this study, a strong association of positive CA12 staining and a positive ER status of primary tumours was seen (data not shown), and the membrane or cytoplasmic CA12 staining of primary tumours did not have a significant association with SLN metastasis.

Lymphatic invasion of tumours is a well-recognised risk factor for lymph node metastasis [3,4,21]. Ugras et al. [3] showed, in a large retrospective cohort including 11,596 patients, that when lymphovascular invasion was absent, the risk of any nodal involvement was 0.28 (95\% CI, 0.26 to 0.31 ) compared to when it was present. As in other studies, lymphatic invasion of primary tumours was one of the strongest risk factors for SLN metastasis in this study.

The Ki-67 labelling index is a surrogate marker for tumour proliferation and thus predictive of a poor treatment outcome in early breast cancer [22]. However, the association between SLN metastasis and the Ki-67 labelling index in early breast cancer is controversial. Bader et al. [23] showed that $\mathrm{Ki}-67 \geq 18 \%$ was associated with axillary lymph node metastases $(18.9 \%$ vs. $40.9 \%, \mathrm{p}<0.001)$ in patients with $\mathrm{T} 1$ breast cancer who underwent level I/II axillary dissection. In contrast, Koyama et al. [24] reported that the Ki-67 labelling index of the primary tumour was significantly lower in SLN positive patients, although Terasaki-Fukuzawa et al. [25] observed no association of Ki-67 labelling index with lymph node metastasis. In this study, similar to Koyama's, SLN metastasis was associated with ER positivity and a low Ki-67 labelling index. In multivariate analysis, low Ki-67 was a significant risk factor for SLN metastasis.

In RSM1 using three risk factors, including lymphatic invasion, the Ki-67 labelling index of primary tumours, and 
expression of CA9 in stromal fibroblasts, the $\mathrm{N}$ stage was associated with the risk score (RS), and the mean number of positive lymph node increased proportional to RS. Of note, in patients without the three risk factors, none of the patients had lymph node metastasis. As the relationship of Ki-67 labelling index with SLN metastasis in other studies is ambivalent [23-25], we evaluated RSM2 using two factors, including lymphatic invasion of primary tumours and CA9 staining of stromal cells (Table 4). In patients without these risk factors, 95\% had negative SLN, suggesting the crucial role of lymphatic invasion of primary tumours and CA9 expression of stromal cells in SLN metastasis.

There are some limitations in this study. There may be undetected confounding factors that affect the results of this study because of the retrospective design. This study includes only patients with early breast cancer in a single centre. Thus, to expand and validate the effect of IHC staining of CA9 and CA12 on SLN metastasis, larger cohorts from several centres are necessary.

\section{Conclusion}

In summary, CA9 staining of stromal cells is a significant risk factor for SLN metastasis in addition to lymphatic invasion and a low Ki-67 labeling index of primary tumours. Patients without these three risk factors did not have SLN metastasis. In patients with neither lymphatic invasion of primary tumours nor CA9 staining of stromal cells, 95\% of patients had negative SLN. IHC staining of CA12 was not a risk factor for SLN metastasis. To validate these results, larger cohort studies are warranted.

\section{Conflicts of Interest}

Conflict of interest relevant to this article was not reported.

\section{Acknowledgments}

This work was supported by a grant No. 11-2012-021 from the SNUBH Research Fund.

\section{References}

1. Sugie T, Sawada T, Tagaya N, Kinoshita T, Yamagami K, Suwa $\mathrm{H}$, et al. Comparison of the indocyanine green fluorescence and blue dye methods in detection of sentinel lymph nodes in early-stage breast cancer. Ann Surg Oncol. 2013;20:2213-8.

2. Veronesi U, Cascinelli N, Mariani L, Greco M, Saccozzi R, Luini A, et al. Twenty-year follow-up of a randomized study comparing breast-conserving surgery with radical mastectomy for early breast cancer. N Engl J Med. 2002;347:1227-32.

3. Ugras S, Stempel M, Patil S, Morrow M. Estrogen receptor, progesterone receptor, and HER2 status predict lymphovascular invasion and lymph node involvement. Ann Surg Oncol. 2014;21:3780-6.

4. Lee JH, Kim SH, Suh YJ, Shim BY, Kim HK. Predictors of axillary lymph node metastases (ALNM) in a Korean population with T1-2 breast carcinoma: triple negative breast cancer has a high incidence of ALNM irrespective of the tumor size. Cancer Res Treat. 2010;42:30-6.

5. Brahimi-Horn MC, Chiche J, Pouyssegur J. Hypoxia and cancer. J Mol Med (Berl). 2007;85:1301-7.

6. Lundgren K, Holm C, Landberg G. Hypoxia and breast cancer: prognostic and therapeutic implications. Cell Mol Life Sci.
2007;64:3233-47

7. Milani M, Harris AL. Targeting tumour hypoxia in breast cancer. Eur J Cancer. 2008;44:2766-73.

8. Shin HJ, Rho SB, Jung DC, Han IO, Oh ES, Kim JY. Carbonic anhydrase IX (CA9) modulates tumor-associated cell migration and invasion. J Cell Sci. 2011;124(Pt 7):1077-87.

9. Wykoff CC, Beasley N, Watson PH, Campo L, Chia SK, English R, et al. Expression of the hypoxia-inducible and tumorassociated carbonic anhydrases in ductal carcinoma in situ of the breast. Am J Pathol. 2001;158:1011-9.

10. McShane LM, Altman DG, Sauerbrei W, Taube SE, Gion M, Clark GM, et al. Reporting recommendations for tumor marker prognostic studies (remark). Exp Oncol. 2006;28: 99-105.

11. Hammond ME, Hayes DF, Wolff AC, Mangu PB, Temin S. American Society of Clinical Oncology/College of American Pathologists guideline recommendations for immunohistochemical testing of estrogen and progesterone receptors in breast cancer. J Oncol Pract. 2010;6:195-7.

12. Wolff AC, Hammond ME, Schwartz JN, Hagerty KL, Allred DC, Cote RJ, et al. American Society of Clinical Oncology / Col- 
lege of American Pathologists guideline recommendations for human epidermal growth factor receptor 2 testing in breast cancer. J Clin Oncol. 2007;25:118-45.

13. Chia SK, Wykoff CC, Watson PH, Han C, Leek RD, Pastorek $\mathrm{J}$, et al. Prognostic significance of a novel hypoxia-regulated marker, carbonic anhydrase IX, in invasive breast carcinoma. J Clin Oncol. 2001;19:3660-8.

14. Aomatsu N, Yashiro M, Kashiwagi S, Kawajiri H, Takashima $\mathrm{T}$, Ohsawa M, et al. Carbonic anhydrase 9 is associated with chemosensitivity and prognosis in breast cancer patients treated with taxane and anthracycline. BMC Cancer. 2014; 14:400.

15. Schutze D, Milde-Langosch K, Witzel I, Rody A, Karn T, Schmidt M, et al. Relevance of cellular and serum carbonic anhydrase IX in primary breast cancer. J Cancer Res Clin Oncol. 2013;139:747-54.

16. Brockton NT, Klimowicz AC, Bose P, Petrillo SK, Konno M, Rudmik L, et al. High stromal carbonic anhydrase IX expression is associated with nodal metastasis and decreased survival in patients with surgically-treated oral cavity squamous cell carcinoma. Oral Oncol. 2012;48:615-22.

17. Fiaschi T, Giannoni E, Taddei ML, Cirri P, Marini A, Pintus G, et al. Carbonic anhydrase IX from cancer-associated fibroblasts drives epithelial-mesenchymal transition in prostate carcinoma cells. Cell Cycle. 2013;12:1791-801.

18. Hsieh MJ, Chen KS, Chiou HL, Hsieh YS. Carbonic anhydrase XII promotes invasion and migration ability of MDA-MB-231 breast cancer cells through the p38 MAPK signaling pathway. Eur J Cell Biol. 2010;89:598-606.
19. Barnett DH, Sheng S, Charn TH, Waheed A, Sly WS, Lin CY, et al. Estrogen receptor regulation of carbonic anhydrase XII through a distal enhancer in breast cancer. Cancer Res. 2008; 68:3505-15.

20. Watson PH, Chia SK, Wykoff CC, Han C, Leek RD, Sly WS, et al. Carbonic anhydrase XII is a marker of good prognosis in invasive breast carcinoma. Br J Cancer. 2003;88:1065-70.

21. Brenin DR, Manasseh DM, El-Tamer M, Troxel A, Schnabel F, Ditkoff BA, et al. Factors correlating with lymph node metastases in patients with T1 breast cancer. Ann Surg Oncol. 2001; 8:432-7.

22. de Azambuja E, Cardoso F, de Castro G Jr, Colozza M, Mano MS, Durbecq V, et al. Ki-67 as prognostic marker in early breast cancer: a meta-analysis of published studies involving 12,155 patients. Br J Cancer. 2007;96:1504-13.

23. Bader AA, Tio J, Petru E, Buhner M, Pfahlberg A, Volkholz H, et al. T1 breast cancer: identification of patients at low risk of axillary lymph node metastases. Breast Cancer Res Treat. 2002; 76:11-7.

24. Koyama Y, Ichikawa H, Sakata J, Sakata E, Tatsuda K, Hasegawa $\mathrm{M}$, et al. The association between sentinel lymph node metastasis and Ki-67 labeling index. Adv Breast Cancer Res. 2013;2:60-5.

25. Terasaki-Fukuzawa Y, Kijima H, Suto A, Takeshita T, Iezumi $\mathrm{K}$, Sato S, et al. Decreased nm23 expression, but not Ki-67 labeling index, is significantly correlated with lymph node metastasis of breast invasive ductal carcinoma. Int J Mol Med. 2002;9:25-9. 